\title{
Frequency of Electrolyte Imbalance in Children of Pediatricacute Diarrhea
}

\section{Muhammad Zain Ul Abadin ${ }^{1}$, Maria Iftikar ${ }^{2}$, Navaira Arshad ${ }^{3}, Z_{\text {Zeeshan Mahmood }}^{4}$, Beenish Tahira, Muhammad Shafiq ${ }^{6}$, Tayaba Khawar Butt ${ }^{7}$}

\begin{abstract}
Objective: To find out the electrolyte abnomalities among children affected by pediatric acute diarrhea.

Methods: Two hundred and eighty children fulfilling the inclusion/exclusion criteria admitted in Department of Pediatrics, Services Hospital, Lahore was taken. Informed consent of the parents of children was obtained to include their data in the study. Demographic profile age, gender, was recorded. Every children with acute diarrhea was followed through $5 \mathrm{cc}$ venous blood test sent to the hospital laboratory with the help of pramedical staff of the hospital for evaluation of electrolyte imbalance.

Results: In our study, out of 280 cases of Pediatric acute diarrhea, $50 \%(n=140)$ had up to 1 year of age. $64 \%(n=174)$ were male while $38 \%(n=106)$ were females. Hyponatremia was the most common electrolyte abnormality effecting $22.5 \%$ males and $6.5 \%$ females followed by hypokalemia.Electrolyte imbalance was more common in children of age group 2-5years.

Conclusion: Electrolyte and acid base abnormalities are common in children with acute watery diarrhea affecting children of 3-12 months of age. Hyponatremia and hypokalemia are the most common abnormalities. Both abnormalities increase with an increase in duration of diarrhea, occur more frequently in patients who are severely dehydrated.
\end{abstract}

Keywords: Electrolyte imbalance, paediatric acute diarrhea, hyponatremia

\section{Introduction}

$\mathrm{D}$ iarrhea is defined as passage of three or more liquid stools per day or increase loss of water and electrolytes in stool leading to imbalance and later deficiency of water and electrolytes in the body. ${ }^{1}$ Childhood diarrhea is the most common cause of morbidity and mortality, especially in the low and middle- income countries. ${ }^{2}$ Acute diarrheal disease is the leading cause of morbidity and mortality in Pakistan.

Each year diarrhea kills around 760, 000 children

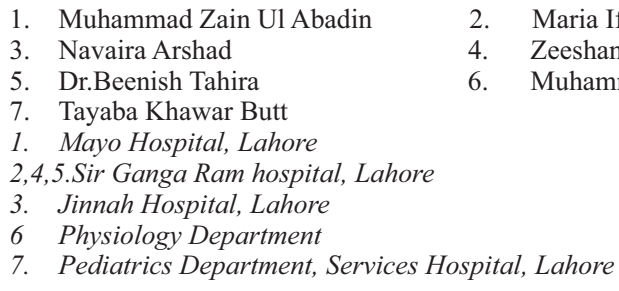

Correspondence

Dr. M. Zain-ul-Abadin

Mayo Hospital Lahore, me nabiha@hotmail.com

$\begin{array}{ll}\text { Submission Date: } & 28-07-2020 \\ \text { 1st Revision Date: } & 10-09-2020\end{array}$

Acceptance Date: 03-11-2020 under five. Globally, there are nearly 1.7 billion cases of diarrheal disease every year. It is the leading cause of malnutrition in children under five years of age. ${ }^{3}$ Short term complications of acute diarrhea are dehydration, electrolyte imbalance, malnutrition, shock and death. ${ }^{4}$

Normal stool has an alkaline $\mathrm{pH}$. Sodium and potassium are the primary stool solutes. The sodium plus potassium concentration in stool usually ranges between 130 and $150 \mathrm{mEq} / \mathrm{L}$. Other cations, such as calcium and magnesium, are present at much lower concentrations. $^{5}$

Commonly observed electrolyte disturbances are hypernatremia, hyponatremia and hypokalemia. ${ }^{6}$ In secretory diarrhea, there is also hyperchloremic metabolic acidosis which can present as hypotension and acute renal failure. Lactic acidosis may supervene as a result of tissue hypoperfusion. ${ }^{7}$ Hyponatremia is one of the most common electrolyte disorders, the frequency ranges from $1 \%$ to $40 \%$ in general hospital population. It can lead to cerebral edema, coma and brainstem herniation if not timely detected and properly treated. $^{8}$ 
Hypernatremia is associated with high mortality rate if sodium concentration exceeds $158 \mathrm{mEq} / 1$. it largely effects central nervous system. ${ }^{9}$ Loss of bicarbonate and potassium leads to acidosis and hypokalemia which can lead to neuromuscular, gastrointestinal and cardiac symptoms. ${ }^{1}$

A study was done in Kathmandu University, they included 57 patients below 15 years of age who presented with diarrhea. Forty six (79\%) children had some form of electrolyte abnormality while rest $11(21 \%)$ patients had normal electrolyte levels. The major electrolyte abnormalities noted were hyponatremia and hypokalaemia. ${ }^{10}$

As it is a common cause of morbidity and mortality in Pakistan, and limited literature is available in reference to electrolyte and acid base imbalance and there is research done on this important issue in my center. Young children die of this simple problem, so my rationale is to highlight the major electrolyte which lead to death from this simple problem.

\section{Methods}

It was a cross sectional study conducted at Department of Pediatrics, Services, Hospital, Lahore which includes 280 cases as calculated with $95 \%$ confidence level, $2 \%$ margin of error and taking expected percentage conservative of hyperkalemia i.e. 3\% (least among all) different electrolytes imbalance among children with acute diarrhea. ${ }^{13}$ sampling was done by Non probability consecutive sampling technique our inclusion criteria was children between 3 months to 5 years of age and children with acute diarrhea (Passage of loose or watery stools at least 3 times in 24 hours, for less than 14 days). Children having any chronic GI illness like celiac disease and congenital adrenal hyperplasia and children having other systemic disease like renal (urea $>20 \mathrm{mg} / 1$, creatinine $>1.3 \mathrm{mg}$ / dl), gastrointestinal and metabolic diseases were excluded from the study because these cases will act as effect modifiers and if included in the study will introduce bias in the result.

\section{Data Collection Procedure}

- Children with acute diarrhea, aged between 3 months to 5 years of age were selected for the study. They were diagnosed on history (i.e. passing loose or watery stools at least 3 times in
24 hours). Source of patients will be through emergency room.

- An informed consent was taken after explaining the benefits of the study. Patients having malnutrition or any other abnormality like renal, respiratory, cardiac, gastrointestinal and metabolic diseases which can affect electrolytes will be excluded from the study. Demographic details like age and sex were noted. History of present illness was inquired for the type, severity and duration of symptoms i.e. loose motions and vomiting. The type of food intake, source of water supply, any medications taken at home and socioeconomic status of the child was noted. All the children were examined with regard to general behavior, temperature, heart rate, respiratory rate, skin turgor, anterior fontanelle and for abdominal distension.

- Under aseptic measures, about 2cc of blood was drawn by $24 \mathrm{G}$ butterfly needle and non-oxalated sample was sent for serum electrolytes; sodium and potassium to the biochemistry laboratory of Services Institute of Medical Sciences, Lahore. Sodium and potassium were measured through ion specific electrode method.

- The collected data was entered and analyzed using SPSS version 16.

- Quantitative variables which includes age in months, number of episodes of diarrhea and electrolyte levels were summarized as mean \pm SD.

- Qualitative variables like sex used were expressed as percentages and proportions.

- The frequency and percentage of children with electrolyte imbalance i.e. hyponatremia, hypernatremia, hypokalemia or hyperkalemia in acute diarrhea was calculated.

- Data was stratified for age, electrolyte imbalance, gender, duration of diarrhea to address the effect of modifiers.

- Chi-square test was used to compare qualitative data

- $\quad \mathrm{p}<0.05$ was considered as significant. 
Hypernatremia is associated with high mortality rate if sodium concentration exceeds $158 \mathrm{mEq} / 1$. it largely effects central nervous system. ${ }^{9}$ Loss of bicarbonate and potassium leads to acidosis and hypokalemia which can lead to neuromuscular, gastrointestinal and cardiac symptoms. ${ }^{1}$

A study was done in Kathmandu University, they included 57 patients below 15 years of age who presented with diarrhea. Forty six (79\%) children had some form of electrolyte abnormality while rest $11(21 \%)$ patients had normal electrolyte levels. The major electrolyte abnormalities noted were hyponatremia and hypokalaemia. ${ }^{10}$

As it is a common cause of morbidity and mortality in Pakistan, and limited literature is available in reference to electrolyte and acid base imbalance and there is research done on this important issue in my center. Young children die of this simple problem, so my rationale is to highlight the major electrolyte which lead to death from this simple problem.

\section{Methods}

It was a cross sectional study conducted at Department of Pediatrics, Services, Hospital, Lahore which includes 280 cases as calculated with $95 \%$ confidence level, $2 \%$ margin of error and taking expected percentage conservative of hyperkalemia i.e. 3\% (least among all) different electrolytes imbalance among children with acute diarrhea. ${ }^{13}$ sampling was done by Non probability consecutive sampling technique our inclusion criteria was children between 3 months to 5 years of age and children with acute diarrhea (Passage of loose or watery stools at least 3 times in 24 hours, for less than 14 days). Children having any chronic GI illness like celiac disease and congenital adrenal hyperplasia and children having other systemic disease like renal (urea $>20 \mathrm{mg} / 1$, creatinine $>1.3 \mathrm{mg}$ / dl), gastrointestinal and metabolic diseases were excluded from the study because these cases will act as effect modifiers and if included in the study will introduce bias in the result.

\section{Data Collection Procedure}

- Children with acute diarrhea, aged between 3 months to 5 years of age were selected for the study. They were diagnosed on history (i.e. passing loose or watery stools at least 3 times in
24 hours). Source of patients will be through emergency room.

- An informed consent was taken after explaining the benefits of the study. Patients having malnutrition or any other abnormality like renal, respiratory, cardiac, gastrointestinal and metabolic diseases which can affect electrolytes will be excluded from the study. Demographic details like age and sex were noted. History of present illness was inquired for the type, severity and duration of symptoms i.e. loose motions and vomiting. The type of food intake, source of water supply, any medications taken at home and socioeconomic status of the child was noted. All the children were examined with regard to general behavior, temperature, heart rate, respiratory rate, skin turgor, anterior fontanelle and for abdominal distension.

- Under aseptic measures, about 2cc of blood was drawn by $24 \mathrm{G}$ butterfly needle and non-oxalated sample was sent for serum electrolytes; sodium and potassium to the biochemistry laboratory of Services Institute of Medical Sciences, Lahore. Sodium and potassium were measured through ion specific electrode method.

- The collected data was entered and analyzed using SPSS version 16.

- Quantitative variables which includes age in months, number of episodes of diarrhea and electrolyte levels were summarized as mean \pm SD.

- Qualitative variables like sex used were expressed as percentages and proportions.

- The frequency and percentage of children with electrolyte imbalance i.e. hyponatremia, hypernatremia, hypokalemia or hyperkalemia in acute diarrhea was calculated.

- Data was stratified for age, electrolyte imbalance, gender, duration of diarrhea to address the effect of modifiers.

- Chi-square test was used to compare qualitative data

- $\quad \mathrm{p}<0.05$ was considered as significant. 
Hypernatremia is associated with high mortality rate if sodium concentration exceeds $158 \mathrm{mEq} / 1$. it largely effects central nervous system. ${ }^{9}$ Loss of bicarbonate and potassium leads to acidosis and hypokalemia which can lead to neuromuscular, gastrointestinal and cardiac symptoms. ${ }^{1}$

A study was done in Kathmandu University, they included 57 patients below 15 years of age who presented with diarrhea. Forty six (79\%) children had some form of electrolyte abnormality while rest $11(21 \%)$ patients had normal electrolyte levels. The major electrolyte abnormalities noted were hyponatremia and hypokalaemia. ${ }^{10}$

As it is a common cause of morbidity and mortality in Pakistan, and limited literature is available in reference to electrolyte and acid base imbalance and there is research done on this important issue in my center. Young children die of this simple problem, so my rationale is to highlight the major electrolyte which lead to death from this simple problem.

\section{Methods}

It was a cross sectional study conducted at Department of Pediatrics, Services, Hospital, Lahore which includes 280 cases as calculated with $95 \%$ confidence level, $2 \%$ margin of error and taking expected percentage conservative of hyperkalemia i.e. 3\% (least among all) different electrolytes imbalance among children with acute diarrhea. ${ }^{13}$ sampling was done by Non probability consecutive sampling technique our inclusion criteria was children between 3 months to 5 years of age and children with acute diarrhea (Passage of loose or watery stools at least 3 times in 24 hours, for less than 14 days). Children having any chronic GI illness like celiac disease and congenital adrenal hyperplasia and children having other systemic disease like renal (urea $>20 \mathrm{mg} / 1$, creatinine $>1.3 \mathrm{mg}$ / dl), gastrointestinal and metabolic diseases were excluded from the study because these cases will act as effect modifiers and if included in the study will introduce bias in the result.

\section{Data Collection Procedure}

- Children with acute diarrhea, aged between 3 months to 5 years of age were selected for the study. They were diagnosed on history (i.e. passing loose or watery stools at least 3 times in
24 hours). Source of patients will be through emergency room.

- An informed consent was taken after explaining the benefits of the study. Patients having malnutrition or any other abnormality like renal, respiratory, cardiac, gastrointestinal and metabolic diseases which can affect electrolytes will be excluded from the study. Demographic details like age and sex were noted. History of present illness was inquired for the type, severity and duration of symptoms i.e. loose motions and vomiting. The type of food intake, source of water supply, any medications taken at home and socioeconomic status of the child was noted. All the children were examined with regard to general behavior, temperature, heart rate, respiratory rate, skin turgor, anterior fontanelle and for abdominal distension.

- Under aseptic measures, about 2cc of blood was drawn by $24 \mathrm{G}$ butterfly needle and non-oxalated sample was sent for serum electrolytes; sodium and potassium to the biochemistry laboratory of Services Institute of Medical Sciences, Lahore. Sodium and potassium were measured through ion specific electrode method.

- The collected data was entered and analyzed using SPSS version 16.

- Quantitative variables which includes age in months, number of episodes of diarrhea and electrolyte levels were summarized as mean \pm SD.

- Qualitative variables like sex used were expressed as percentages and proportions.

- The frequency and percentage of children with electrolyte imbalance i.e. hyponatremia, hypernatremia, hypokalemia or hyperkalemia in acute diarrhea was calculated.

- Data was stratified for age, electrolyte imbalance, gender, duration of diarrhea to address the effect of modifiers.

- Chi-square test was used to compare qualitative data

- $\quad \mathrm{p}<0.05$ was considered as significant. 
College of Physicians and Surgeons Pakistan 2016; 26(5): 394-98.

14. Alam N H, Hamadani J D, Dewan N, Fuchs G J. Efficacy and safety of a modified oral rehydration solution (ReSoMaL) in the treatment of severely malnourished children with watery diarrhea. Pediatr. 2003; 143(5):614-9.

15. Molla AM, Rahman M, Sarker SA, Sack DA, Molla A. Stool electrolyte content and purging rates in diarrhea caused by rotavirus, enterotoxigenic E. coli, and V. cholerae in children. J Pediatr 1981; 98:835-8.

16. Asma B, Mohammad A S, Nighat H. Frequency of hyponatraemia and hypokalaemia in malnourished children with acute diarrhoea. J Pak Med Assoc 2016; 66: 1077-9.
17. Kamberi T H, Azemi M, Avdiu M, Jaha V I, Uka V G. Malnourished Children with Acute Diarrhea. Arch Dis Child 2012;97(2):302724.0675.

18. Uysal G, Sokmen A, Vidinlisan S. Clinical risk factors for fatal diarrhea in hospitalized children. Ind J Paedtr 2000; 67: 329-33.

19. Agarwal R, Afzalpurkar R. Pathophysiology of potassium absorption and secretion by the human intestine. Gastroenterology 1994; 107: 548-71.

20. Morisky DE, Kar SB, Chaudhry AS, Chen KR, Shaheen M, Chickering K. Update on ORS Usage in Pakistan: Results of a National Study. Pakistan Journal of Nutrition.2002; 1(3):143-50. 